\title{
THE MILNOR INVARIANTS OF CLOVER LINKS
}

\author{
KODAI WADA AND AKIRA YASUHARA
}

\begin{abstract}
J.P. Levine introduced a clover link to investigate the indeterminacy of the Milnor invariants of a link. It is shown that for a clover link, the Milnor numbers of length at most $2 k+1$ are well-defined if those of length at most $k$ vanish, and that the Milnor numbers of length at least $2 k+2$ are not well-defined if those of length $k+1$ survive. For a clover link $c$ with the Milnor numbers of length at most $k$ vanishing, we show that the Milnor number $\mu_{c}(I)$ for a sequence $I$ is well-defined up to the greatest common devisor of $\mu_{c}(J)^{\prime} s$, where $J$ is a subsequence of $I$ obtained by removing at least $k+1$ indices. Moreover, if $I$ is a non-repeated sequence with length $2 k+2$, the possible range of $\mu_{c}(I)$ is given explicitly. As an application, we give an edge-homotopy classification of 4-clover links.
\end{abstract}

\section{INTRODUCTION}

The Milnor invariant introduced by J. Milnor [7, 8]. For an oriented ordered $n$-component link $L$ in the 3 -sphere $S^{3}$ with peripheral information, the Milnor number $\mu_{L}(I)$, which is an integer, is specified by a finite sequence $I$ in $\{1,2, \ldots, n\}$. The Milnor $\bar{\mu}$-invariant $\bar{\mu}_{L}(I)$ is the residue class of $\mu_{L}(I)$ modulo the greatest common devisor of $\mu_{L}(J)$ 's, where $J$ is obtained from proper subsequence of $I$ by permuting cyclicly. The length of the sequence $I$ is called the length of $\bar{\mu}_{L}(I)$ and denoted by $|I|$. His original definition of the Milnor invariant eliminates the indeterminacy of the possible variations of the Milnor numbers caused by different choices of peripheral elements.

In [6, J. P. Levine examined the Milnor invariants from the point of view of based links, in order to understand the indeterminacy. A based link is a link for which some peripheral information is specified, i.e., meridians (the weakest) or both meridians and longitudes (the strongest). It is known that these invariants are completely well-defined for the strongest form of basing (disk links [5] or string links [2]). As basing only slightly stronger than the specification of meridians, he introduced an $n$-clover link which is an embedded graph consisting of $n$ loops, each loop connected to a vertex by an edge in $S^{3}$. The Milnor number of a clover link is defined up to flatly isotopy and it is shown that those of length $\leq k$ vanish implies those of length $\leq 2 k+1$ are completely non-indeterminate [6].

The first author [10] redefined the Milnor number of a clover link up to ambient isotopy as follows. Given an $n$-clover link $c$, we construct an $n$-component bottom tangle $\gamma\left(F_{c}\right)$ by using a disk/band surface $F_{c}$ of $c$. In [6], Levine defined the Milnor number of a bottom tangle. Therefore we define the Milnor number $\mu_{c}$ of an $n$ clover link $c$ to be the Milnor number $\mu_{\gamma\left(F_{c}\right)}$. (In [6], a bottom tangle is called a string link. The name 'bottom tangle' follows K. Habiro 3.) In [10, it is shown that the same result as Levine [6] holds while there are infinitely many choices of $\gamma\left(F_{c}\right)$ for $c$.

Date: January 14, 2021

The second author is partially supported by a JSPS Grant-in-Aid for Scientific Research (C) (\#26400081). 
Unfortunately, the Milnor numbers of length at least $2 k+2$ are not well-defined if those of length $k+1$ survive. In this paper, we show that the Milnor number $\mu_{c}(I)$ of a sequence $I$ modulo $\delta_{c}^{k}(I)$ is well-defined if the Milnor numbers of length $\leq k$ vanish, where $\delta_{c}^{k}(I)$ is the greatest common devisor of $\mu_{c}(J)^{\prime} s$, where $J$ is range to over all subsequences of $I$ obtained by removing at least $k+1$ indices. In fact, we have the following theorem.

Theorem 1.1. Let $c$ be an $n$-clover link and $l_{c}$ a link which is the disjoint union of loops of $c$. If the Milnor numbers of $l_{c}$ for sequences with length $\leq k$ vanish, then the residue class of $\mu_{c}(I)$ modulo $\delta_{c}^{k}(I)$ is an invariant for any sequence $I$.

Remark 1.2. In contrast to the $\bar{\mu}$-invariant for a link in $S^{3}$, we do not need to take cyclic permutation for getting $\delta_{c}^{k}(I)$.

It is an important property that for non-repeated sequences, the Milnor invariants of links are link-homotopy invariants [7. The first author showed that the Milnor numbers for any non-repeated sequence with length $\leq 3$ give an edge-homotopy classification of 3-clover links [10, where edge-homotopy 9 is an equivalence relation, which is a generalization of link-homotopy, generated by crossing changes on the same spatial edge. We also discuss giving an edge-homotopy classification of 4-clover links. The Milnor numbers for non-repeated sequences with length 4 could be useful to have an edge-homotopy classification of 4-clover links. But they are not well-defined in general. Hence we consider the set of all Milnor numbers of length 4 for all disk/band surfaces of $c$. More generally, we define the following set;

$$
H_{c}(2 k+2, j)=\left\{\sum_{S \in \mathcal{S}_{j}^{2 k+1}} \mu_{\gamma\left(F_{c}\right)}(S j) X_{S} \mid F_{c}: \text { a disk/band surface of c }\right\}
$$

for each integer $j(1 \leq j \leq n)$, where $\mathcal{S}_{j}^{2 k+1}$ is the set of length- $(2 k+1)$ nonrepeated sequences without containing $j$ and for a sequence $S=i_{1} i_{2} \ldots i_{2 k+1}$, $X_{S}=X_{i_{1}} X_{i_{2}} \cdots X_{i_{2 k+1}}$ is a monomial in non-commutative variables $X_{1}, \ldots, X_{n}$. Since $H_{c}(2 k+2, j)$ consists of the Milnor numbers $\mu_{\gamma\left(F_{c}\right)}(S j)\left(S \in \mathcal{S}_{j}^{2 k+1}\right)$ for all disk/band surfaces of $c$, it is an invariant of $c$. While it seems too big to handle $H_{c}(2 k+2, j)$, we have the following theorem.

Theorem 1.3. Let $c$ be an n-clover link and $F_{c}$ a disk/band surface of $c$. If the Milnor numbers of $l_{c}$ for non-repeated sequences with length $\leq k$ vanish, then we have the following:

$$
\left.\begin{array}{l}
\left\{\sum _ { \substack { H | = | I | = k \\
J I l \in \mathcal { S } _ { j } ^ { 2 k + 1 } } } \mu _ { \gamma ( F _ { c } ) } ( J j ) \mu _ { \gamma ( F _ { c } ) } ( I l ) \left(\sum_{i_{s} \in\{J\}} m_{l i_{s}} X_{J_{<s}}\left(X_{i_{s} I l}-X_{i_{s} l I}-X_{I l i_{s}}+X_{l I i_{s}}\right) X_{J_{s<}}\right.\right. \\
\left.\quad+m_{l j}\left(X_{I l J}-X_{l I J}-X_{J I l}+X_{J l I}\right)\right)+\sum_{S \in \mathcal{S}_{j}^{2 k+1}} \mu_{\gamma\left(F_{c}\right)}(S j) X_{S} \mid m_{p q}=m_{q p} \in \mathbb{Z}
\end{array}\right\},
$$

where for a sequence $J=i_{1} \ldots i_{m},\{J\}=\left\{i_{1}, \ldots, i_{m}\right\}$ and $J_{<s}\left(\right.$ resp. $\left.J_{s<}\right)$ is a subsequence $i_{1} \ldots i_{s-1}$ (resp. $\left.i_{s+1} \ldots i_{m}\right)$ of $J$ for $1 \leq s \leq m$, and $X_{J_{<1}}$ and $X_{J_{m<}}$ are defined to be 1 .

This theorem implies that the set $H_{c}(2 k+2, j)$ is obtained from the Milnor numbers of $\gamma\left(F_{c}\right)$ for any single disk/band surface $F_{c}$ of $c$, that is, $H_{c}(2 k+2, j)$ is specified explicitly. 
By the following corollary we have that $H_{c}(2 k+2, j)$ is not only an invariant of $c$ but also an edge-homotopy invariant.

Corollary 1.4. For a clover link $c$, if the Milnor numbers of $l_{c}$ for non-repeated sequences with length $\leq k$ vanish, then $H_{c}(2 k+2, j)$ is an edge-homotopy invariant of $c$.

It is the definition that the Milnor numbers of length 1 are zero. If $k=1$, then the theorem above holds without the condition. The following example follows directly from Theorem 1.3 .

Example 1.5. For a 4-clover link $c$ and a disk/band surface $F_{c}$ of $c$, we have

$$
\begin{aligned}
H_{c}(4,4)= & \left\{\left(\begin{array}{c}
\mu_{\gamma\left(F_{c}\right)}(14) \mu_{\gamma\left(F_{c}\right)}(23)\left(m_{13}-m_{34}-m_{12}+m_{24}\right) \\
+\mu_{\gamma\left(F_{c}\right)}(12) \mu_{\gamma\left(F_{c}\right)}(34)\left(m_{24}-m_{23}-m_{14}+m_{13}\right)
\end{array}\right) X_{123}\right. \\
& +\left(\begin{array}{c}
\mu_{\gamma\left(F_{c}\right)}(14) \mu_{\gamma\left(F_{c}\right)}(23)\left(m_{34}-m_{13}-m_{24}+m_{12}\right) \\
+\mu_{\gamma\left(F_{c}\right)}(13) \mu_{\gamma\left(F_{c}\right)}(24)\left(m_{34}-m_{23}-m_{14}+m_{12}\right)
\end{array}\right) X_{132} \\
& +\left(\begin{array}{c}
\mu_{\gamma\left(F_{c}\right)}(13) \mu_{\gamma\left(F_{c}\right)}(24)\left(m_{23}-m_{34}-m_{12}+m_{14}\right) \\
+\mu_{\gamma\left(F_{c}\right)}(12) \mu_{\gamma\left(F_{c}\right)}(34)\left(m_{23}-m_{24}-m_{13}+m_{14}\right)
\end{array}\right) X_{213} \\
& +\left(\begin{array}{c}
\mu_{\gamma\left(F_{c}\right)}(14) \mu_{\gamma\left(F_{c}\right)}(23)\left(m_{34}-m_{13}-m_{24}+m_{12}\right) \\
+\mu_{\gamma\left(F_{c}\right)}(13) \mu_{\gamma\left(F_{c}\right)}(24)\left(m_{34}-m_{23}-m_{14}+m_{12}\right)
\end{array}\right) X_{231} \\
& +\left(\begin{array}{c}
\mu_{\gamma\left(F_{c}\right)}(13) \mu_{\gamma\left(F_{c}\right)}(24)\left(m_{23}-m_{34}-m_{12}+m_{14}\right) \\
+\mu_{\gamma\left(F_{c}\right)}(12) \mu_{\gamma\left(F_{c}\right)}(34)\left(m_{23}-m_{24}-m_{13}+m_{14}\right)
\end{array}\right) X_{312} \\
+ & \left(\begin{array}{l}
\mu_{\gamma\left(F_{c}\right)}(14) \mu_{\gamma\left(F_{c}\right)}(23)\left(m_{13}-m_{34}-m_{12}+m_{24}\right) \\
+\mu_{\gamma\left(F_{c}\right)}(12) \mu_{\gamma\left(F_{c}\right)}(34)\left(m_{24}-m_{23}-m_{14}+m_{13}\right)
\end{array}\right) X_{321} \\
+ & \left.\sum_{S \in \mathcal{S}_{4}^{3}} \mu_{\gamma\left(F_{c}\right)}(S 4) X_{S} \mid m_{p q} \in \mathbb{Z}\right\} .
\end{aligned}
$$

This together with the theorem below gives us an edge-homotopy classification of 4-clover links, see Remark 1.7.

Theorem 1.6. Let $c$ and $c^{\prime}$ be 4-clover links. They are edge-homotopic if and only if $H_{c}(4,4) \cap H_{c^{\prime}}(4,4) \neq \emptyset$ and $\mu_{c}(I)=\mu_{c^{\prime}}(I)$ for any non-repeated sequence $I$ with $|I| \leq 3$.

Remark 1.7. By Example 1.5, we are able to determine whether $H_{c}(4,4) \cap H_{c^{\prime}}(4,4)$ is empty or not. Hence by combining Example 1.5 and Theorem 1.6, we obtain an edge-homotopy classification of 4-clover links.

\section{The Milnor numbers of ClOver LinkS}

In this section we define the Milnor numbers for clover links.

2.1. A construction of bottom tangles. An $n$-component tangle is a properly embedded disjoint union of $n$ arcs in the 3-cube $[0,1]^{3}$. An $n$-component bottom tangle $\gamma=\gamma_{1} \cup \gamma_{2} \cup \cdots \cup \gamma_{n}$ defined by Levine [6] is a tangle with $\partial \gamma_{i}=$ $\left\{\left(\frac{2 i-1}{2 n+1}, \frac{1}{2}, 0\right),\left(\frac{2 i}{2 n+1}, \frac{1}{2}, 0\right)\right\} \subset \partial[0,1]^{3}$ for each $i(=1,2, \ldots, n)$.

A spatial graph is an embedded graph in $S^{3}$. Let $C_{n}$ be a graph consisting of $n$ oriented loops $e_{1}, e_{2}, \ldots, e_{n}$, each loop $e_{i}$ connected to a vertex $v$ by an edge $f_{i}(i=1,2, \ldots, n)$, see Figure 2.1. An $n$-clover link in $S^{3}$ is a spatial graph of $C_{n}$ [6]. The each part of a clover link corresponding to $e_{i}, f_{i}$ and $v$ of $C_{n}$ are called the leaf, stem and root, denoted by the same notations respectively. 


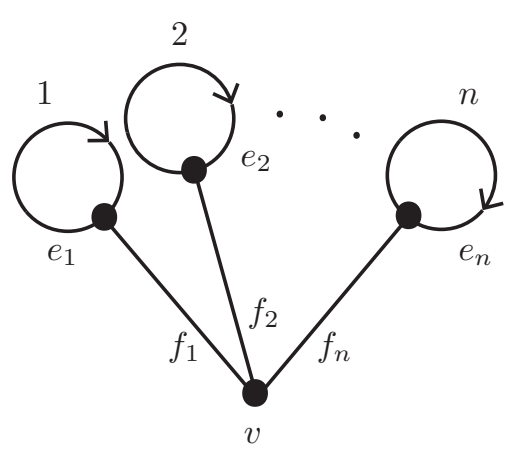

FiguRE 2.1. the graph $C_{n}$

L. Kauffman, J. Simon, K. Wolcott and P. Zhao 4 defined disk/band surfaces of spatial graphs. For a spatial graph $\Gamma$, a disk/band surface $F_{\Gamma}$ of $\Gamma$ is a compact, oriented surface in $S^{3}$ such that $\Gamma$ is a deformation retract of $F_{\Gamma}$ contained in the interior of $F_{\Gamma}$. Note that any disk/band surface of a spatial graph is ambient isotopic to a surface constructed by putting a disk at each vertex of the spatial graph, connecting the disks with bands along the spatial edges. We remark that for a spatial graph, there are infinitely many disk/band surfaces up to ambient isotopy.

Given an $n$-clover link, we construct an $n$-component bottom tangle using a disk/band surface of the clover link as follows:

(1) For an $n$-clover link $c$, let $F_{c}$ be a disk/band surface of $c$ and let $D$ be a disk which contains the root. From now on, we may assume that the intersection $D \cap \bigcup_{i=1}^{n} f_{i}$ and orientations of the disks are as illustrated in Figure 2.2.

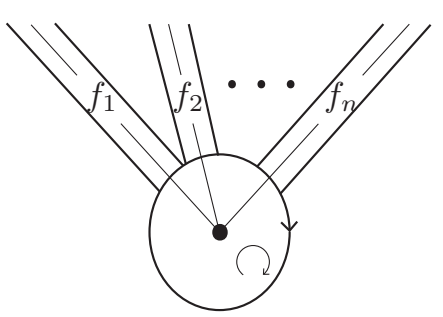

D

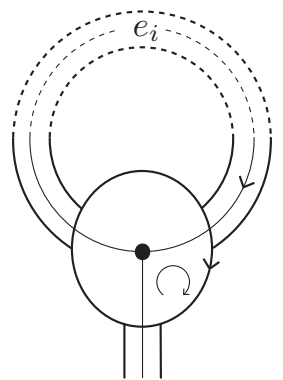

FigURE 2.2 .

(2) Let $N(D)$ be the regular neighborhood of $D$ and $N(D)$ the interior of $N(D)$. Since $S^{3} \backslash N(D)$ is homeomorphic to the 3-ball, $F_{c} \backslash N(D)$ can be seen as a disjoint union of surfaces in the 3-ball. Hence $\partial F_{c} \backslash N(D)$ is a disjoint union of $n$-arcs and $n$-circles $\bigcup_{i=1}^{n} S_{i}^{1}$ in the 3 -ball.

(3) Since the 3 -ball is homeomorphic to $[0,1]^{3}$, we obtain an oriented ordered $n$-component bottom tangle $\gamma\left(F_{c}\right)$ from $\left(\partial F_{c} \backslash N(D)\right) \backslash \bigcup_{i=1}^{n} S_{i}^{1}$ as illustrated in (3) and (4) of Figure 2.3. We call $\gamma\left(F_{c}\right)$ an $n$-component bottom tangle obtained from $F_{c}$. 


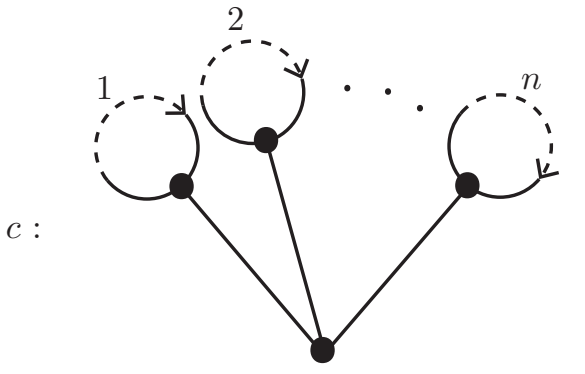

(1)

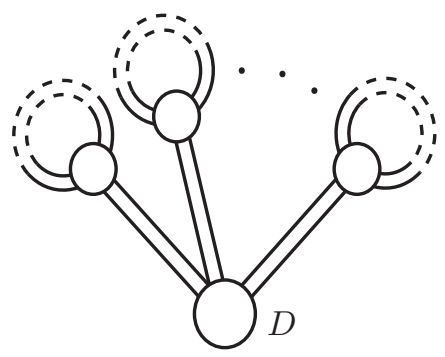

$(2)$

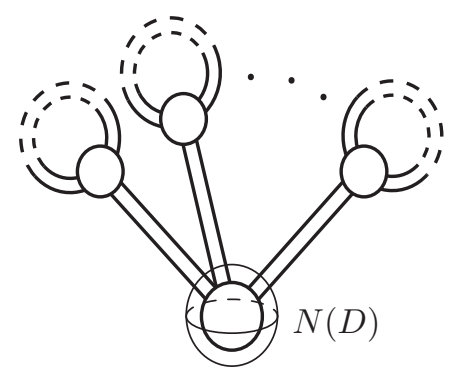

(3)

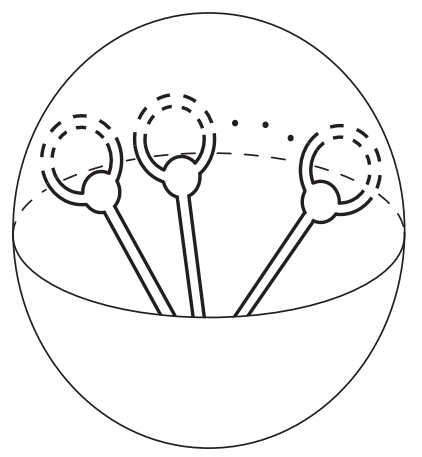

$\partial F_{c} \backslash N(D)$

(4)

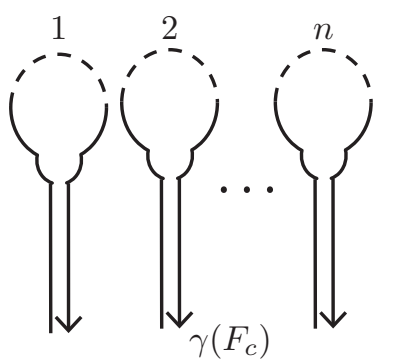

Figure 2.3. A method for obtaining a bottom tangle from a diskband surface of a clover link

While there are infinitely many disk/band surfaces of a clover link which satisfy the condition (1) above, they are related by certain local moves as follows.

Lemma 2.1. 10, Proposition 2.5] For an n-clover link c, any two disk/band surfaces $F_{c}$ and $F_{c}^{\prime}$ are transformed into each other by adding full-twists to bands (Figure 2.4 (a)) and a single move illustrated in Figure 2.4 (b).

(a)
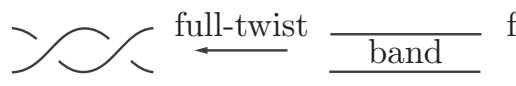
$\stackrel{\text { full-twist }}{\longrightarrow}$

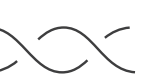

(b)
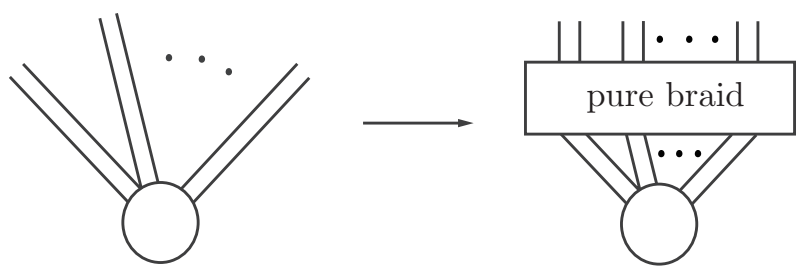

Figure 2.4. Two local moves of disk/band surfaces 
2.2. Milnor invariants. Let us briefly recall from [6] the definition of the Milnor number of a bottom tangle. Let $\gamma=\gamma_{1} \cup \gamma_{2} \cup \cdots \cup \gamma_{n}$ be an oriented ordered $n$ component bottom tangle in $[0,1]^{3}$ with $\partial \gamma_{i}=\left\{\left(\frac{2 i-1}{2 n+1}, \frac{1}{2}, 0\right),\left(\frac{2 i}{2 n+1}, \frac{1}{2}, 0\right)\right\} \subset \partial[0,1]^{3}$ for each $i(=1,2, \ldots, n)$. Let $G$ be the fundamental group of $[0,1]^{3} \backslash \gamma$ with a base point $p=\left(\frac{1}{2}, 0,0\right)$ and $G_{q}$ the $q$ th lower central subgroup of $G$. Let $\alpha_{i}$ and $\lambda_{i}$ be the $i$ th meridian and $i$ th longitude of $\gamma$ respectively as illustrated in Figures 2.5 We assume that $\lambda_{i}$ is trivial in $G / G_{2}$. Since the quotient group $G / G_{q}$ is generated by $\alpha_{1}, \alpha_{2}, \ldots, \alpha_{n}$ [1], $\gamma$ is represented by $\alpha_{1}, \alpha_{2}, \ldots, \alpha_{n}$ modulo $G_{q}$.
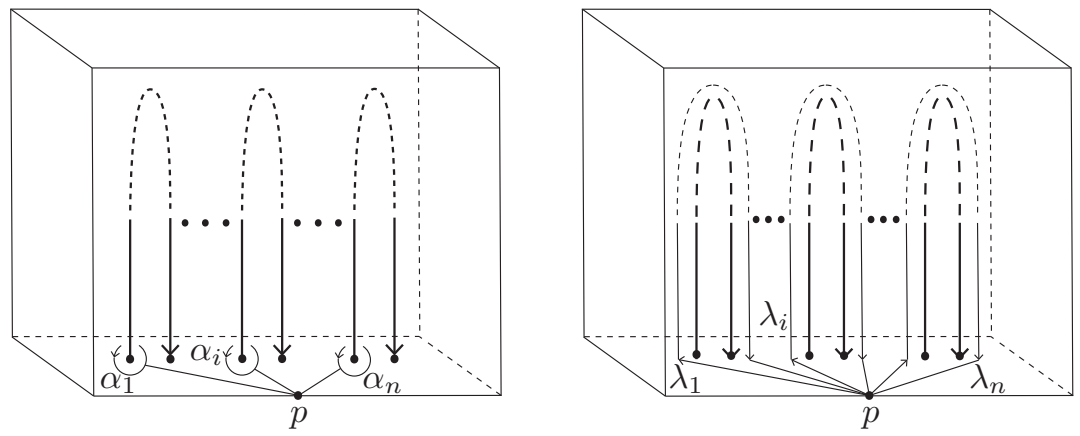

Figure 2.5. meridians and longitudes

We consider the Magnus expansion of $\lambda_{j}$. The Magnus expansion is a homomorphism (denoted $E$ ) from a free group $\left\langle\alpha_{1}, \alpha_{2}, \ldots, \alpha_{n}\right\rangle$ to the formal power series ring in non-commutative variables $X_{1}, X_{2}, \ldots, X_{n}$ with integer coefficients defined as follows. $E\left(\alpha_{i}\right)=1+X_{i}, E\left(\alpha_{i}^{-1}\right)=1-X_{i}+X_{i}^{2}-X_{i}^{3}+\cdots(i=1,2, \ldots, n)$.

For a sequence $I=i_{1} i_{2} \ldots i_{k-1} j\left(i_{m} \in\{1,2, \ldots, n\}, k \leq q\right)$, we define the Milnor number $\mu_{\gamma}(I)$ to be the coefficient of $X_{i_{1} i_{2} \cdots i_{k-1}}$ in $E\left(\lambda_{j}\right)$ (we define $\mu_{\gamma}(j)=$ 0 ), which is an invariant [6]. (In [6], the set of $\lambda_{i}$ 's, without taking the Magnus expansion, is called the Milnor's $\bar{\mu}$-invariant.) For a bottom tangle $\gamma=\gamma_{1} \cup \gamma_{2} \cup$ $\cdots \cup \gamma_{n}$, an oriented link $L(\gamma)=L_{1} \cup L_{2} \cup \cdots \cup L_{n}$ in $S^{3}$ can be defined by $L_{i}=\gamma_{i} \cup a_{i}$, where $a_{i}$ is a line segment connecting $\left(\frac{2 i-1}{2 n+1}, \frac{1}{2}, 0\right)$ and $\left(\frac{2 i}{2 n+1}, \frac{1}{2}, 0\right)$, see Figure 2.6. We call $L(\gamma)$ the closure of $\gamma$. On the other hand, for any oriented link

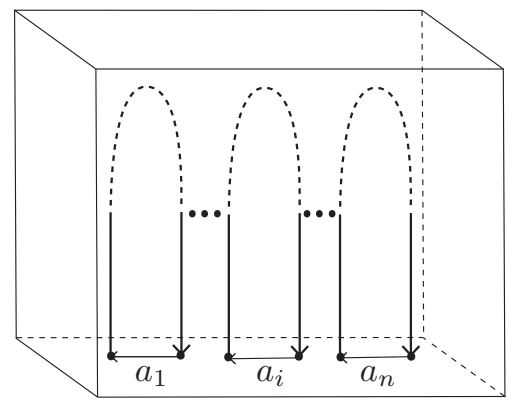

FIGURE 2.6 .

$L$ in $S^{3}$, there is a bottom tangle $\gamma_{L}$ such that the closure of $\gamma_{L}$ is equal to $L$. So we define the Milnor number of $L$ to be the Milnor number of $\gamma_{L}$. Let $\Delta_{L}(I)$ be the greatest common devisor of $\mu_{L}(J)^{\prime} s$, where $J$ is obtained from proper subsequence of $I$ by permuting cyclicly. The Milnor invariant $\bar{\mu}_{L}(I)$ is the residue class of $\mu_{L}(I)$ modulo $\Delta_{L}(I)$. We note that for a sequence $I$, if we have $\Delta_{L}(I)=0$, then the Milnor invariant $\bar{\mu}_{L}(I)$ is equal to the Milnor number $\mu_{\gamma_{L}}(I)$. Now we define the Milnor number of a clover link. 
Definition 2.2. Let $c$ be an n-clover link and $F_{c}$ a disk/band surface of $c$. Let $\gamma\left(F_{c}\right)$ be the n-component bottom tangle obtained from $F_{c}$. For a sequence $I$, the Milnor number $\mu_{c}(I)$ of $c$ is defined to be the Milnor number $\mu_{\gamma\left(F_{c}\right)}(I)$.

Remark 2.3. While $\mu_{c}(I)$ depends on a choice of $F_{c}$, the first author [10] proved the following result: Let $l_{c}$ be a link which is the disjoint union of leaves of $c$. If the Milnor numbers of $l_{c}$ for sequences with length $\leq k$ vanish, then $\mu_{c}(I)$ is well-defined for any sequence $I$ with $|I| \leq 2 k+1$.

\section{Proof of Theorem 1.1}

In this section we will give a proof of Theorem 1.1 .

An $n$-component tangle $u=u_{1} \cup u_{2} \cup \cdots \cup u_{n}$ is an $n$-component string link if for each $i(=1,2, \ldots, n)$, the boundary $\partial u_{i}=\left\{\left(\frac{2 i-1}{2 n+1}, \frac{1}{2}, 0\right),\left(\frac{2 i-1}{2 n+1}, \frac{1}{2}, 1\right)\right\} \subset \partial[0,1]^{3}$. In particular, $u$ is trivial if for each $i(=1,2, \ldots, n), u_{i}=\left\{\left(\frac{2 i-1}{2 n+1}, \frac{1}{2}\right)\right\} \times[0,1]$ in $[0,1]^{3}$.

Here we introduce a $S L$-move [10] given by a string link $u$ which is a transformation of an $n$-component bottom tangle $\gamma=\gamma_{1} \cup \gamma_{2} \cup \cdots \cup \gamma_{n}$ with $\partial \gamma_{i}=$ $\left\{\left(\frac{2 i-1}{2 n+1}, \frac{1}{2}, 0\right),\left(\frac{2 i}{2 n+1}, \frac{1}{2}, 0\right)\right\} \subset \partial[0,1]^{3}$.

(1) Let $u=u_{1} \cup u_{2} \cup \cdots \cup u_{n}$ be an oriented ordered $n$-component string link in $[0,1]^{3}$. For each $i(=1,2, \ldots, n)$, we consider an arc $u_{i}^{\prime}$ which is parallel to the $i$ th component $u_{i}$ of $u$ with opposite orientation and $\partial u_{i}^{\prime}=$ $\left\{\left(\frac{2 i}{2 n+1}, \frac{1}{2}, 0\right),\left(\frac{2 i}{2 n+1}, \frac{1}{2}, 1\right)\right\} \subset \partial[0,1]^{3}$, see Figure 3.7 .

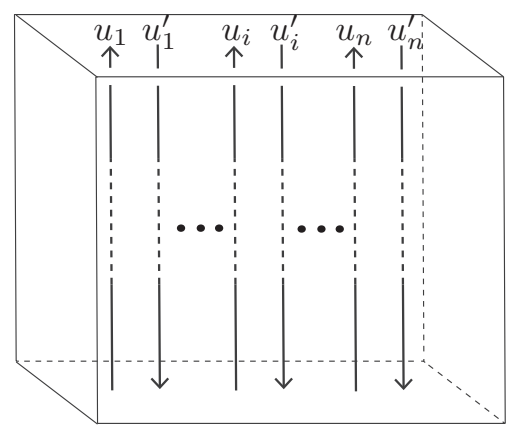

FiguRE 3.7.

(2) Let $\gamma^{\prime}=\gamma_{1}^{\prime} \cup \gamma_{2}^{\prime} \cup \cdots \cup \gamma_{n}^{\prime}$ be an $n$-component bottom tangle in $[0,1]^{3}$ defined by

$$
\gamma_{i}^{\prime}=h_{0}\left(u_{i} \cup u_{i}^{\prime}\right) \cup h_{1}\left(\gamma_{i}\right)
$$

for $i=1,2, \ldots, n$, where $h_{0}, h_{1}:([0,1] \times[0,1]) \times[0,1] \rightarrow([0,1] \times[0,1]) \times[0,1]$ are embeddings defined by

$$
h_{0}(x, t)=\left(x, \frac{1}{2} t\right) \text { and } h_{1}(x, t)=\left(x, \frac{1}{2}+\frac{1}{2} t\right)
$$

for $x \in([0,1] \times[0,1])$ and $t \in[0,1]$.

We say that $\gamma^{\prime}$ is obtained from $\gamma$ by a SL-move. For example, see Figure 3.8 We note that if $u$ is trivial, a SL-move is just adding full-twists or nothing. A SL-move is determined by a string link and a number of full-twists, that is, 'SL' stands for String Link.

Proposition 3.1. Let $\gamma$ be an n-component bottom tangle and $\gamma^{\prime}$ a bottom tangle obtained from $\gamma$ by a SL-move. If the Milnor numbers of $\gamma$ and $\gamma^{\prime}$ for sequences with length $\leq k$ vanish, then for any sequence $I$,

$$
\mu_{\gamma^{\prime}}(I) \equiv \mu_{\gamma}(I) \quad \bmod \delta_{\gamma}^{k}(I)
$$

where $\delta_{\gamma}^{k}(I)$ is the greatest common devisor of $\mu_{\gamma}(J)^{\prime}$ s for a proper subsequence $J$ of $I$ which is obtained by removing at least $k+1$ indices. 

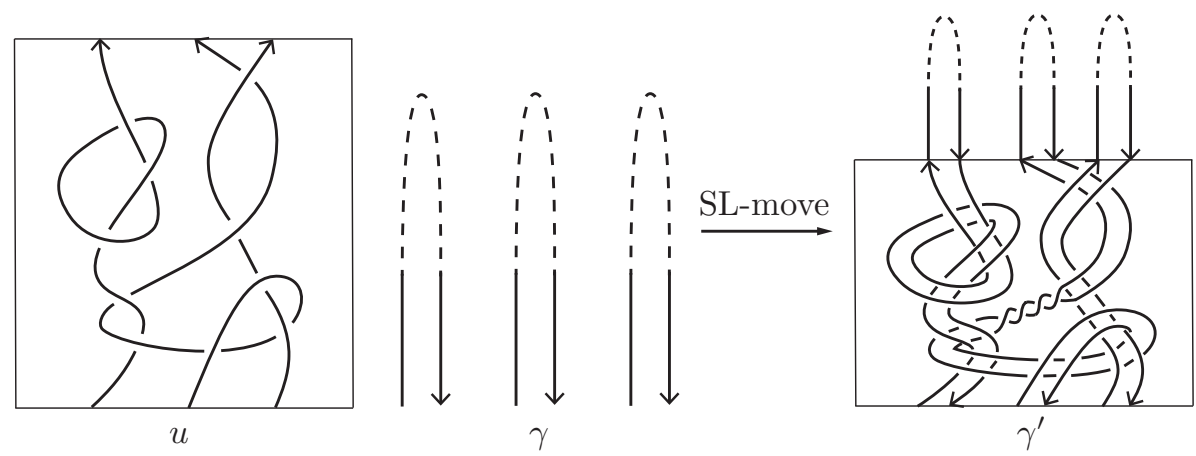

Figure 3.8. An example of a SL-move

Proof. The proof is by induction on the length $|I|=q$. If $q \leq k$, the proposition clearly holds. We note that, by the induction hypothesis, $\delta_{\gamma}^{k}(I)=\delta_{\gamma^{\prime}}^{k}(I)$. Denote respectively by $\alpha_{i}, \lambda_{i}$ (resp. $\alpha_{i}^{\prime}, \lambda_{i}^{\prime}$ ) the $i$ th meridian and $i$ th longitude of $\gamma$ (resp. $\gamma^{\prime}$ ) for $1 \leq i \leq n$. Let $E_{X}$ (resp. $E_{Y}$ ) be the Magnus expansion in non-commutative variables $X_{1}, \ldots, X_{n}$ (resp. $Y_{1}, \ldots, Y_{n}$ ) obtained by replacing $\alpha_{i}$ by $1+X_{i}$ (resp. $\alpha_{i}^{\prime}$ by $\left.1+Y_{i}\right)$ for $1 \leq i \leq n$. Fix $j$, by the assumption, the Milnor numbers for $\gamma$ and $\gamma^{\prime}$ of length $\leq k$ vanish, so $E_{X}\left(\lambda_{j}\right)$ and $E_{Y}\left(\lambda_{j}^{\prime}\right)$ can be written respectively in the form

$$
E_{X}\left(\lambda_{j}\right)=1+F_{j}(X) \text { and } E_{Y}\left(\lambda_{j}^{\prime}\right)=1+F_{j}^{\prime}(Y),
$$

where $F_{j}(X)\left(=F_{j}\left(X_{1}, \ldots, X_{n}\right)\right)$ and $F_{j}^{\prime}(Y)\left(=F_{j}^{\prime}\left(Y_{1}, \ldots, Y_{n}\right)\right)$ are terms of degree $\geq k$.

Here we define a set of polynomials;

$D_{j}^{k}=\left\{\begin{array}{l|l}\sum \nu\left(i_{1} \ldots i_{m}\right) Y_{i_{1}} \ldots Y_{i_{m}} & \begin{array}{l}\nu\left(i_{1} \ldots i_{m}\right) \equiv 0 \\ \nu\left(i_{1} \ldots i_{m}\right) \in \mathbb{Z},\end{array} \quad \bmod \delta_{\gamma}^{k}\left(i_{1} \ldots i_{m} j\right), \begin{array}{l}m<q \\ m \geq q\end{array}\end{array}\right\}$.

Then it is enough to show

$$
F_{j}^{\prime}(Y)-F_{j}(Y) \in D_{j}^{k} .
$$

The following claims are shown by similar to the assertions (16) and (18) in 8 .

Claim 1. $D_{j}^{k}$ is a two-sided ideal of the formal power series ring in non-commutative variables $Y_{1}, \ldots, Y_{n}$ with integer coefficients.

Claim 2. If at least $k$ variables are inserted anywhere in a term $\mu\left(i_{1} i_{2} \ldots i_{m} j\right) Y_{i_{1} i_{2} \ldots i_{m}}$, then the resulting term belongs to $D_{j}^{k}$.

Let $u_{i}$ be the $i$ th longitude of a string link which gives the SL-move, see Figure 3.9. In the proof of [10, Lemma 2.6], it is shown that the degree of each term in $E_{Y}\left(u_{i}^{ \pm 1}\right)-1$ is at least $k+1$. Set $E_{Y}\left(u_{i}\right)=1+G_{i}(Y)$ and $E_{Y}\left(u_{i}^{-1}\right)=1+\overline{G_{i}}(Y)$, where $G_{i}(Y)$ and $\overline{G_{i}}(Y)$ mean the terms of degree $\geq k+1$. Since $\alpha_{i}=u_{i}^{-1} \alpha_{i}^{\prime} u_{i}$ (where $\alpha_{i}$ is assumed to be an element of $\pi_{1}\left([0,1]^{3} \backslash \gamma^{\prime}\right)$ ), we have

$$
\begin{aligned}
E_{Y}\left(\alpha_{i}\right) & =E_{Y}\left(u_{i}^{-1} \alpha_{i}^{\prime} u_{i}\right) \\
& =\left(1+\overline{G_{i}}(Y)\right)\left(1+Y_{i}\right)\left(1+G_{i}(Y)\right) \\
& =\left(1+\overline{G_{i}}(Y)\right)\left(1+G_{i}(Y)\right)+\left(1+\overline{G_{i}}(Y)\right) Y_{i}\left(1+G_{i}(Y)\right) \\
& =1+Y_{i}+Y_{i} G_{i}(Y)+\overline{G_{i}}(Y) Y_{i}+\overline{G_{i}}(Y) Y_{i} G_{i}(Y) .
\end{aligned}
$$

Hence $E_{Y}\left(\lambda_{j}\right)$ is obtained from $E_{X}\left(\lambda_{j}\right)$ by substituting $X_{i}$ for

$$
Y_{i}+Y_{i} G_{i}(Y)+\overline{G_{i}}(Y) Y_{i}+\overline{G_{i}}(Y) Y_{i} G_{i}(Y) .
$$

Set $E_{Y}\left(\lambda_{j}\right)=1+H_{j}(Y)$, where $H_{j}(Y)$ is the terms of degree $\geq k$. Note that terms of degree $\leq 2 k$ of $H_{j}(Y)-F_{j}(Y)$ vanish, and that any term of $H_{j}(Y)-F_{j}(Y)$ of 


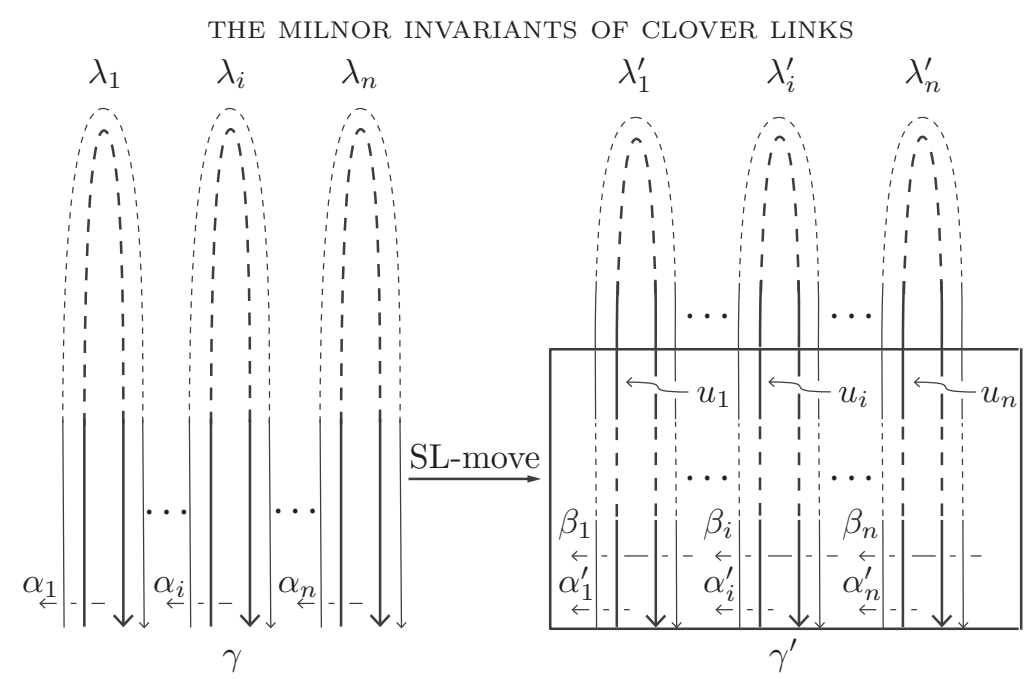

FiguRE 3.9.

degree $\geq 2 k+1$ is obtained from $F_{j}(Y)$ by inserting at least $k+1$ variables. By Claim 2,

$$
H_{j}(Y)-F_{j}(Y) \in D_{j}^{k}
$$

Since $\lambda_{j}^{\prime}=u_{j} \lambda_{j} u_{j}^{-1}$ (where $\lambda_{j}$ is assumed to be an element of $\pi_{1}\left([0,1]^{3} \backslash \gamma^{\prime}\right)$ ),

$$
\begin{aligned}
E_{Y}\left(\lambda_{j}^{\prime}\right) & =E_{Y}\left(u_{j} \lambda_{j} u_{j}^{-1}\right) \\
& =\left(1+G_{j}(Y)\right)\left(1+H_{j}(Y)\right)\left(1+\overline{G_{j}}(Y)\right) \\
& =1+H_{j}(Y)+H_{j}(Y) \overline{G_{j}}(Y)+G_{j}(Y) H_{j}(Y)+G_{j}(Y) H_{j}(Y) \overline{G_{j}}(Y) .
\end{aligned}
$$

It follows from Claims 1 and 2 that we have

$$
F_{j}^{\prime}(Y)-H_{j}(Y)=H_{j}(Y) \overline{G_{j}}(Y)+G_{j}(Y) H_{j}(Y)+G_{j}(Y) H_{j}(Y) \overline{G_{j}}(Y) \in D_{j}^{k} .
$$

Since $H_{j}(Y)-F_{j}(Y) \in D_{j}^{k}$, by Claim 1, we have

$$
F_{j}^{\prime}(Y)-F_{j}(Y) \in D_{j}^{k} .
$$

This completes the proof.

Proof of Theorem 1.1. By Lemma 2.1, any two disk/band surfaces $F_{c}$ and $F_{c}^{\prime}$ of an $n$-component clover link $c$ are transformed into each other by the moves (a) and (b) illustrated in Figure 2.4 So two bottom tangles $\gamma\left(F_{c}\right)$ and $\gamma\left(F_{c}^{\prime}\right)$ are transformed into each other by a SL-move. Since the both closures $L\left(\gamma\left(F_{c}\right)\right)$ and $L\left(\gamma\left(F_{c}^{\prime}\right)\right)$ are ambient isotopic to $l_{c}$, by the hypothesis of Theorem 1.1.

$$
0=\bar{\mu}_{l_{c}}(J)=\mu_{\gamma\left(F_{c}\right)}(J)=\mu_{\gamma\left(F_{c}^{\prime}\right)}(J)
$$

for any sequence $J$ with $|J| \leq k$. Hence by Proposition 3.1

$$
\mu_{\gamma\left(F_{c}^{\prime}\right)}(I) \equiv \mu_{\gamma\left(F_{c}\right)}(I) \quad \bmod \delta_{\gamma\left(F_{c}\right)}^{k}(I)
$$

for any sequence $I$. This completes the proof.

\section{Proof of Theorems 1.3 and 1.6}

In this section we will give proofs of Theorems 1.3 1.6 and Corollary 1.4

Proposition 4.1. Let $\gamma$ be an n-component bottom tangle and $\gamma^{\prime}$ a bottom tangle obtained from $\gamma$ by a SL-move which is given by a string link $u$. If the Milnor numbers of $\gamma$ and $\gamma^{\prime}$ for non-repeated sequences with length $\leq k$ vanish, then we have the following: 


$$
\begin{aligned}
& \sum_{S \in \mathcal{S}_{j}^{2 k+1}}\left(\mu_{\gamma^{\prime}}(S j)-\mu_{\gamma}(S j)\right) Y_{S} \\
= & \sum_{\substack{|J|=|I|=k \\
J I l \in \mathcal{S}_{j}^{2 k+1}}} \mu_{\gamma}(J j) \mu_{\gamma}(I l) \sum_{i_{s} \in\{J\}} \mu_{u}\left(l i_{s}\right) Y_{J_{<s}}\left(Y_{i_{s} I l}-Y_{i_{s} l I}-Y_{I l i_{s}}+Y_{l I i_{s}}\right) Y_{J_{s<}} \\
& +\sum_{\substack{|J|=|I|=k \\
J I l \in \mathcal{S}_{j}^{2 k+1}}} \mu_{\gamma}(J j) \mu_{\gamma}(I l) \mu_{u}(l j)\left(Y_{I l J}-Y_{l I J}-Y_{J I l}+Y_{J l I}\right) .
\end{aligned}
$$

Proof. We compare with the Magnus expansions of the $j$ th longitudes of $\gamma$ and $\gamma^{\prime}$. Denote respectively by $\alpha_{i}, \lambda_{i}$ (resp. $\alpha_{i}^{\prime}, \lambda_{i}^{\prime}$ ) the $i$ th meridian and $i$ th longitude of $\gamma\left(\right.$ resp. $\gamma^{\prime}$ ) for $1 \leq i \leq n$. Let $E_{X}$ (resp. $E_{Y}$ ) be the Magnus expansion in non-commutative variables $X_{1}, \ldots, X_{n}$ (resp. $Y_{1}, \ldots, Y_{n}$ ) obtained by replacing $\alpha_{i}$ by $1+X_{i}\left(\right.$ resp. $\alpha_{i}^{\prime}$ by $\left.1+Y_{i}\right)$ for $1 \leq i \leq n$. By the assumption, the Minor numbers for $\gamma$ and $\gamma^{\prime}$ of degree $k$ coincide, hence denote by

$$
E_{X}\left(\lambda_{j}\right)=1+\sum_{I \in \mathcal{S}_{j}^{k}} \mu_{\gamma}(I j) X_{I}+r_{j}(X)+\mathcal{O}_{X}(2)
$$

and

$$
E_{Y}\left(\lambda_{j}^{\prime}\right)=1+\sum_{I \in \mathcal{S}_{j}^{k}} \mu_{\gamma}(I j) Y_{I}+r_{j}^{\prime}(Y)+\mathcal{O}_{Y}(2),
$$

where $r_{j}(X)$ and $r_{j}^{\prime}(Y)$ mean the terms of degree $\geq k+1$ and $\mathcal{O}_{X}(2)$ (resp. $\left.\mathcal{O}_{Y}(2)\right)$ denotes the terms which contain $X_{i}$ (resp. $Y_{i}$ ) at least 2 times for some $i(=1,2, \ldots, n)$. Let $f_{j}(X)=\sum_{I \in \mathcal{S}_{j}^{k}} \mu_{\gamma}(I j) X_{I}$ and $f_{j}(Y)=\sum_{I \in \mathcal{S}_{j}^{k}} \mu_{\gamma}(I j) Y_{I}$.

Let $u_{i}$ be the $i$ th longitude of $u$ and let $\beta_{i}=\left[\lambda_{i}^{\prime}, \alpha_{i}^{\prime}\right]=\lambda_{i}^{\prime-1} \alpha_{i}^{\prime-1} \lambda_{i}^{\prime} \alpha_{i}^{\prime}$, see Figure 3.9. Let $E_{Z}$ be the Magnus expansion in non-commutative variables $Z_{1}, \ldots, Z_{n}$ obtained by replacing $\beta_{i}$ by $1+Z_{i}$ for $1 \leq i \leq n$. Then we have

$$
E_{Z}\left(u_{i}\right)=1+\sum_{l \neq i} \mu_{u}(l i) Z_{l}+\mathcal{O}(2) .
$$

First, we observe $E_{Y}\left(\beta_{l}\right)$ and $E_{Y}\left(\alpha_{i}\right)$, where $\alpha_{i}$ is assumed to be an element of $\pi_{1}\left([0,1]^{3} \backslash \gamma^{\prime}\right)$. Since $E_{Y}\left(\lambda_{l}^{\prime}\right) E_{Y}\left(\lambda_{l}^{\prime-1}\right)=1$, set $E_{Y}\left(\lambda_{l}^{\prime-1}\right)=1-f_{l}(Y)+\overline{r_{l}^{\prime}}(Y)+\mathcal{O}_{Y}(2)$, where $\overline{r_{l}^{\prime}}(Y)$ is the terms of degree $\geq k+1$. Observe that

$$
\begin{aligned}
& E_{Y}\left(\beta_{l}\right) \\
& =E_{Y}\left(\lambda_{l}^{\prime-1} \alpha_{l}^{\prime-1} \lambda_{l}^{\prime} \alpha_{l}^{\prime}\right) \\
& =\left(1-f_{l}(Y)+\overline{r_{l}^{\prime}}(Y)+\mathcal{O}_{Y}(2)\right)\left(1-Y_{l}+\mathcal{O}_{Y}(2)\right)\left(1+f_{l}(Y)+r_{l}^{\prime}(Y)+\mathcal{O}_{Y}(2)\right)\left(1+Y_{l}\right) \\
& =1+f_{l}(Y) Y_{l}-Y_{l} f_{l}(Y)+\mathcal{O}(k+2)+\mathcal{O}_{Y}(2) \\
& =1+\sum_{I \in \mathcal{S}_{l}^{k}} \mu_{\gamma}(I l) Y_{I} Y_{l}-Y_{l} \sum_{I \in \mathcal{S}_{l}^{k}} \mu_{\gamma}(I l) Y_{I}+\mathcal{O}(k+2)+\mathcal{O}_{Y}(2) \\
& =1+\sum_{I \in \mathcal{S}_{l}^{k}} \mu_{\gamma}(I l)\left(Y_{I l}-Y_{l I}\right)+\mathcal{O}(k+2)+\mathcal{O}_{Y}(2) .
\end{aligned}
$$

This implies that $E_{Y}\left(u_{i}\right)$ is obtained from $E_{Z}\left(u_{i}\right)$ by substituting $Z_{l}$ for

$$
\sum_{I \in \mathcal{S}_{l}^{k}} \mu_{\gamma}(I l)\left(Y_{I l}-Y_{l I}\right)+\mathcal{O}(k+2)+\mathcal{O}_{Y}(2)
$$

So we have

$$
E_{Y}\left(u_{i}\right)=1+\sum_{l \neq i} \mu_{u}(l i) \sum_{I \in \mathcal{S}_{l}^{k}} \mu_{\gamma}(I l)\left(Y_{I l}-Y_{l I}\right)+\mathcal{O}(k+2)+\mathcal{O}_{Y}(2) .
$$


Let $g_{i}(Y)=\sum_{l \neq i} \mu_{u}(l i) \sum_{I \in \mathcal{S}_{l}^{k}} \mu_{\gamma}(I l)\left(Y_{I l}-Y_{l I}\right)$. Then we have

$$
E_{Y}\left(u_{i}^{-1}\right)=1-g_{i}(Y)+\mathcal{O}(k+2)+\mathcal{O}_{Y}(2)
$$

Since $\alpha_{i}=u_{i}^{-1} \alpha_{i}^{\prime} u_{i}$, we have

$$
\begin{aligned}
& E_{Y}\left(\alpha_{i}\right) \\
& =E_{Y}\left(u_{i}^{-1} \alpha_{i}^{\prime} u_{i}\right) \\
& =\left(1-g_{i}(Y)+\mathcal{O}(k+2)+\mathcal{O}_{Y}(2)\right)\left(1+Y_{i}\right)\left(1+g_{i}(Y)+\mathcal{O}(k+2)+\mathcal{O}_{Y}(2)\right) \\
& =1+Y_{i}+Y_{i} g_{i}(Y)-g_{i}(Y) Y_{i}+\mathcal{O}(k+3)+\mathcal{O}_{Y}(2) \\
& =1+Y_{i}+\sum_{l \neq i} \mu_{u}(l i) \sum_{I \in \mathcal{S}_{l}^{k}} \mu_{\gamma}(I l)\left(Y_{i I l}-Y_{i l I}-Y_{I l i}+Y_{l I i}\right)+\mathcal{O}(k+3)+\mathcal{O}_{Y}(2) .
\end{aligned}
$$

Now we consider the difference $d_{j}(Y)=E_{Y}\left(\lambda_{j}\right)-\left(1+f_{j}(Y)+r_{j}(Y)+\mathcal{O}_{Y}(2)\right)$. Since $E_{Y}\left(\lambda_{j}\right)$ is obtained from $E_{X}\left(\lambda_{j}\right)\left(=1+f_{j}(X)+r_{j}(X)+\mathcal{O}_{X}(2)\right)$ by substituting $X_{i}$ for

$$
Y_{i}+\sum_{l \neq i} \mu_{u}(l i) \sum_{I \in \mathcal{S}_{l}^{k}} \mu_{\gamma}(I l)\left(Y_{i I l}-Y_{i l I}-Y_{I l i}+Y_{l I i}\right)+\mathcal{O}(k+3)+\mathcal{O}_{Y}(2),
$$

all terms of degree $\leq 2 k$ of $d_{j}(Y)-\mathcal{O}_{Y}(2)$ vanish. The terms of degree $2 k+1$ in $d_{j}(Y)-\mathcal{O}_{Y}(2)$ is obtained from $f_{j}(Y)$ by substituting $Y_{i}$ for

$$
\sum_{l \neq i} \mu_{u}(l i) \sum_{I \in S_{l}^{k}} \mu_{\gamma}(I l)\left(Y_{i I l}-Y_{i l I}-Y_{I l i}+Y_{l I i}\right)
$$

for some $i \in\{1,2, \ldots, n\}$. It follows that

$$
\begin{aligned}
& d_{j}(Y)-\left(\mathcal{O}_{Y}(2)+\mathcal{O}(2 k+2)+\mathcal{O}_{Y_{j}}\right) \\
& =\sum_{J \in \mathcal{S}_{j}^{k}} \mu_{\gamma}(J j) \sum_{i_{s} \in\{J\}} Y_{J_{<s}}\left(\sum_{l \neq i_{s}} \mu_{u}\left(l i_{s}\right) \sum_{J I l \in \mathcal{S}_{j}^{2 k+1}} \mu_{\gamma}(I l)\left(Y_{i_{s} I l}-Y_{i_{s} l I}-Y_{I l i_{s}}+Y_{l I i_{s}}\right)\right) Y_{J_{s<}} \\
& =\sum_{\substack{|J|=|I|=k \\
J I l \in \mathcal{S}_{j}^{2 k+1}}} \mu_{\gamma}(J j) \mu_{\gamma}(I l) \sum_{i_{s} \in\{J\}} \mu_{u}\left(l i_{s}\right) Y_{J_{<s}}\left(Y_{i_{s} I l}-Y_{i_{s} l I}-Y_{I l i_{s}}+Y_{l I i_{s}}\right) Y_{J_{s<}},
\end{aligned}
$$

where $\mathcal{O}_{Y_{j}}$ means the terms which contain $Y_{j}$ at least one time.

Finally, we observe the difference $E_{Y}\left(\lambda_{j}^{\prime}\right)-\left(1+f_{j}(Y)+r_{j}(Y)+\mathcal{O}_{Y}(2)\right)$. Since $\lambda_{j}^{\prime}=u_{j} \lambda_{j} u_{j}^{-1}$ (where $\lambda_{j}$ is assumed to be an element of $\pi_{1}\left([0,1]^{3} \backslash \gamma^{\prime}\right)$ ), we have

$$
\begin{aligned}
& E_{Y}\left(\lambda_{j}^{\prime}\right) \\
& =E_{Y}\left(u_{j} \lambda_{j} u_{j}^{-1}\right) \\
& =1+\left(1+g_{j}(Y)\right)\left(f_{j}(Y)+r_{j}(Y)+d_{j}(Y)\right)\left(1-g_{j}(Y)\right)+\mathcal{O}(2 k+2)+\mathcal{O}_{Y}(2) \\
& =1+f_{j}(Y)+r_{j}(Y)+d_{j}(Y)+g_{j}(Y) f_{j}(Y)-f_{j}(Y) g_{j}(Y)+\mathcal{O}(2 k+2)+\mathcal{O}_{Y}(2) .
\end{aligned}
$$

So we have

$$
\begin{aligned}
& E_{Y}\left(\lambda_{j}^{\prime}\right)-\left(1+f_{j}(Y)+r_{j}(Y)+\mathcal{O}_{Y}(2)\right) \\
& =d_{j}(Y)+\sum_{\substack{|J|=|I|=k \\
J I l \in \mathcal{S}_{j}^{2 k+1}}} \mu_{\gamma}(J j) \mu_{\gamma}(I l) \mu_{u}(l j)\left(Y_{I l J}-Y_{l I J}-Y_{J I l}+Y_{J l I}\right) \\
& +\mathcal{O}(2 k+2)+\mathcal{O}_{Y}(2) \\
& =\sum_{\substack{|J|=|I|=k \\
J I l \in \mathcal{S}_{j}^{2 k+1}}} \mu_{\gamma}(J j) \mu_{\gamma}(I l) \sum_{i_{s} \in\{J\}} \mu_{u}\left(l i_{s}\right) Y_{J_{<s}}\left(Y_{i_{s} I l}-Y_{i_{s} l I}-Y_{I l i_{s}}+Y_{l I i_{s}}\right) Y_{J_{s<}} \\
& +\sum_{\substack{|J|=|I|=k \\
J I l \in \mathcal{S}_{j}^{2 k+1}}} \mu_{\gamma}(J j) \mu_{\gamma}(I l) \mu_{u}(l j)\left(Y_{I l J}-Y_{l I J}-Y_{J I l}+Y_{J l I}\right) \\
& +\mathcal{O}(2 k+2)+\mathcal{O}_{Y}(2)+\mathcal{O}_{Y_{j}} .
\end{aligned}
$$


This completes the proof.

Proof of Theorem 1.3. By Lemma 2.1, any two disk/band surfaces $F_{c}$ and $F_{c}^{\prime}$ of an $n$-component clover link $c$ are transformed into each other by the moves (a) and (b) in Figure 2.4. So two bottom tangles $\gamma\left(F_{c}\right)$ and $\gamma\left(F_{c}^{\prime}\right)$ are transformed into each other by a SL-move. Since the both closures $L\left(\gamma\left(F_{c}\right)\right)$ and $L\left(\gamma\left(F_{c}^{\prime}\right)\right)$ are ambient isotopic to $l_{c}$ and the hypothesis of Theorem 1.3 .

$$
0=\bar{\mu}_{l_{c}}(J)=\mu_{\gamma\left(F_{c}\right)}(J)=\mu_{\gamma\left(F_{c}^{\prime}\right)}(J)
$$

for any sequence $J$ with $|J| \leq k$. Since $\mu_{u}(p q)$ is the 'linking number' of the $p$ th component and the $q$ th component of $u, \mu_{u}(p q)=\mu_{u}(q p)$ and the set

$$
\left\{\mu_{u}(p q) \mid u: \text { a string link }\right\}=\mathbb{Z}
$$

for any $p$ and $q$. This and Proposition 4.1 give us the Thorem 1.3

In order to prove Corollary 1.4 and Theorem [1.6, we need the following lemma given in [10].

Lemma 4.2. [10, Lemma 4.1] Two n-clover links $c$ and $c^{\prime}$ are edge-homotopic if and only if there exist disk/band surfaces $F_{c}$ and $F_{c^{\prime}}$ of $c$ and $c^{\prime}$ respectively such that the two bottom tangles $\gamma\left(F_{c}\right)$ and $\gamma\left(F_{c^{\prime}}\right)$ are link-homotopic.

Proof of Corollary 1.4. Let $c$ and $c^{\prime}$ be $n$-clover links. We assume that they are edge-homotopic. By Lemma 4.2, there exist disk/band surfaces $F_{c}$ and $F_{c^{\prime}}$ of $c$ and $c^{\prime}$ respectively such that $\gamma\left(F_{c}\right)$ and $\gamma\left(F_{c^{\prime}}\right)$ are link-homotopic. This implies that $\mu_{\gamma\left(F_{c}\right)}(I)=\mu_{\gamma\left(F_{c^{\prime}}\right)}(I)$ for any non-repeated sequence $I$ [7], [2]. On the other hand, by Theorem 1.3 the set $H_{c}(2 k+2, j)$ (resp. $\left.H_{c^{\prime}}(2 k+2, j)\right)$ is obtained from the Milnor numbers of $\gamma\left(F_{c}\right)$ (resp. $\gamma\left(F_{c^{\prime}}\right)$ ) for $F_{c}$ (resp. $F_{c^{\prime}}$ ). Hence we have $H_{c}(2 k+2, j)=H_{c^{\prime}}(2 k+2, j)$.

Proof of Theorem 1.6. Suppose that two 4-clover links $c$ and $c^{\prime}$ are edge-homotopic. By Corollary 1.4, $H_{c}(4,4)=H_{c^{\prime}}(4,4)(\neq \emptyset)$. By Lemma 4.2 there exist disk/band surfaces $F_{c}$ and $F_{c^{\prime}}$ such that $\gamma\left(F_{c}\right)$ and $\gamma\left(F_{c^{\prime}}\right)$ are link-homotopic. This implies that the Milnor numbers of $\gamma\left(F_{c}\right)$ and $\gamma\left(F_{c^{\prime}}\right)$ are equal for any non-repeated sequence [7, 2]. Since the Milnor numbers of length $\leq 3$ are always well-defined for clover links (see Remark 2.3), we have $\mu_{c}(I)=\mu_{c^{\prime}}(I)$ for any non-repeated sequence $I$ with $|I| \leq 3$.

Conversely if $H_{c}(4,4) \cap H_{c^{\prime}}(4,4) \neq \emptyset$, then there exist disk/band surfaces $F_{c}$ and $F_{c^{\prime}}$ of $c$ and $c^{\prime}$ respectively such that

$$
\sum_{S \in \mathcal{S}_{4}^{3}} \mu_{\gamma\left(F_{c}\right)}(S 4) X_{S}=\sum_{S \in \mathcal{S}_{4}^{3}} \mu_{\gamma\left(F_{c^{\prime}}\right)}(S 4) X_{S} .
$$

In particular,

$$
\mu_{\gamma\left(F_{c}\right)}(1234)=\mu_{\gamma\left(F_{c^{\prime}}\right)}(1234) \text { and } \mu_{\gamma\left(F_{c}\right)}(2134)=\mu_{\gamma\left(F_{c^{\prime}}\right)}(2134) .
$$

According to the link-homotopy classification theorem for string links by N. Habegger and X. S. Lin [2], for two 4-component string links (bottom tangles) that have common values of the Milnor numbers for non-repeated sequences with length $\leq 3$, they are link-homotopic if and only if their Milnor numbers for sequences 1234 and 2134 coincide, see also [11, Theorem 4.3]. This together with the hypothesis implies that $\gamma\left(F_{c}\right)$ and $\gamma\left(F_{c^{\prime}}\right)$ are link-homotopic. Therefore $c$ and $c^{\prime}$ are edge-homotopic by Lemma 4.2. This completes the proof. 


\section{REFERENCES}

[1] K. T. Chen, Commutator calculus and link invariants, Proc. Amer. Math. Soc. 3 (1952), $44-55$.

[2] N. Habegger; X. S. Lin, The classification of links up to link-homotopy, J. Amer. Math. Soc. 3 (1990), 389-419.

[3] K. Habiro, Bottom tangles and universal invariants, Algebr. Geom. Topol. 6 (2006), 11131214.

[4] L. Kauffman; J. Simon; K. Wolcott; P. Zhao, Invariants of theta-curves and other graphs in 3-space, Topology Appl. 49 (1993), 193-216.

[5] J. LeDimet, Cobordisme d'enlacements de disques (French), Mém. Soc. Math. France (N.S.) No. 32 (1988).

[6] J. P. Levine, The $\bar{\mu}$-invariants of based links, Differential topology (Siegen, 1987), 87-103, Lecture Notes in Math., 1350, Springer, Berlin, (1988).

[7] J. Milnor, Link groups, Ann. of Math. (2) 59 (1954), 177-195.

[8] J. Milnor, Isotopy of links, Algebraic geometry and topology, A symposium in honor of S. Lefschetz, pp. 280-306. Princeton University Press, Princeton, N. J. (1957).

[9] K. Taniyama, Cobordism, homotopy and homology of graphs in $\mathbf{R}^{3}$, Topology 33 (1994), 509-523.

[10] K. Wada, Milnor invariants and edge-homotopy classification of clover links, preprint (2015).

[11] A. Yasuhara, Self delta-equivalence for links whose Milnor's isotopy invariants vanish, Trans. Amer. Math. Soc. 361 (2009), 4721-4749.

Department of Mathematics, School of Education, Waseda University, Nishi-Waseda 1-6-1, Shinjuku-Ku, TOKYO, 169-8050, JAPAN

Department of Mathematics, Tokyo Gaugei University, 4-1-1 Nukuikita-machi, KoganeiSHI, TOKYO, 184-8501, JAPAN

E-mail address: k.wada@akane.waseda.jp, yasuhara@u-gakugei.ac.jp 